\title{
Requirements for a Method of Software Accessibility Conformity Assessment
}

\author{
Fernando Alonso, José L. Fuertes, Ángel L. González, and Loïc Martínez \\ School of Computing, Technical University of Madrid, \\ Campus de Montegancedo, 28660-Boadilla del Monte, Madrid, Spain
}

\begin{abstract}
Given the current trend of public procurement of accessible products and services, including software, there is a need for the suppliers to demonstrate that a software product conforms to accessibility requirements. This is called software accessibility conformity assessment. Today, the evaluation process, and the techniques and tools required to do this, is not as well defined as it is in other contexts, such as web accessibility. Based on our experience in evaluating accessibility, this paper outlines a set of requirements for a method of software accessibility conformity assessment. These requirements will apply across the four conformity assessment functions: selection, determination, review and attestation, and, finally, surveillance.
\end{abstract}

Keywords: Software accessibility, conformity assessment, accessibility evaluation.

\section{Introduction}

There is an increasing demand for accessible software, driven especially by public policies on the public procurement of accessible products and services in the field of information and communication technologies (ICT). Some examples of public procurement policies are Section 508 of the United States' Rehabilitation Act and the European Commission's Mandate M376 on standards supporting accessibility requirements for public procurement

On this point, it is helpful to be able to define what "accessible software" means. There are international and national standards that do this. For example, the upcoming international standard ISO 9241-171 defines accessible software requirements and recommendations. These requirements and recommendations are applicable to the accessibility of interactive systems and they address a wide range of software. They promote increased usability of systems for a wider range of users.

In this context, it is extremely important to evaluate the extent to which software product accessibility conforms to the standards that are promoted by law in many countries. We will use the term "conformity assessment" to denote this idea, applying the ISO definition : demonstration that specified requirements relating to a product, process, system, person or body are fulfilled. This implies that "software accessibility conformity assessment" is the demonstration that accessibility requirements relating to software products are met. 
Unfortunately, the evaluation process, techniques and tools required to perform software accessibility conformity assessments are not as well defined as they are in the web context . Whereas there are plenty of traditional user-based usability evaluation methods, which are good for evaluating effectiveness, efficiency and satisfaction during software product use, an alternative approach is needed for accessibility conformity assessment. The experience from web accessibility assessment indicates that some form of expert-based inspection and testing is better in this case, as discussed in .

To do this, it is essential to have a rigorous method for performing software accessibility conformity assessments to help public administrations in the process of public procurement. In ISO terms, this method is called "conformity assessment scheme", and should include rules, procedures and management activities.

A related issue is tool support. Note that most software accessibility requirements and recommendations cannot be automatically evaluated. This requires the participation of an expert human evaluator. Even so, we believe that tool support is extremely important for (1) supporting the global assessment process and (2) facilitating human evaluation of specific requirements and recommendations.

The rest of the paper is structured as follows. Section 2 deals with software accessibility standards to be used as requirements for conformity assessments. Section 3 describes related work on the evaluation of software accessibility. Section 4 presents the identified requirements for a software accessibility conformity assessment method. Finally, section 5 presents some conclusions and future work.

\section{Software Accessibility Standards}

There are several national and international standards in the field of ICT products and services accessibility. Some, such as the Spanish UNE 139802:2003 and the upcoming ISO 9241-171, are software-specific standards.

Accessibility is strongly related to the concept of usability. In fact, the ISO 9241171 definition of accessibility states that accessibility is the "usability of a product, service, environment or facility by people with the widest range of capabilities".

The ISO 9241-171 requirements and recommendations are divided into four sections: general guidelines (labels, names, user preferences, accessibility adjustments, operation control, compatibility with assistive technologies, closed systems), inputs (alternative input functions, keyboard focus, keyboard input, pointing devices), outputs (general output, visual output, text and fonts, colour, window appearance and behaviour, audio output, text equivalents of audio, media and tactile outputs) and documentation (on-line documentation, help and support services).

This software accessibility standard can be considered to be software's equivalent of the well-known recommendations developed by the World Wide Web Consortium's Web Accessibility Initiative for the web context.

\section{Software Accessibility Assessment}

It would appear to be possible to deal with software accessibility assessment in a similar way to user interface evaluation. According to Nielsen , the four basic ways 
of evaluating user interfaces are: automatically, empirically, formally, and informally. Looking at the current state of the art, there are no fully automatic methods, and formal methods are very difficult to apply and do not scale up well to handle larger user interfaces. Additionally, not all of the user interface evaluation methods are directly applicable for software accessibility conformity assessments. For instance, user tests are a common choice for evaluating accessibility, but this approach cannot demonstrate that a set of accessibility requirements are met.

Other more appropriate methods are based on guidelines or checklists, but they, too, come up against difficulties. Traditionally, there have been three checklist-based ways of determining the accessibility of applications

1) An assistive technology can be executed while the application to be validated is running. This approach is valid for the software case, but requires additional evaluation to cover the full set of accessibility requirements.

2) Inspection tools can be used. They report the values of predetermined sets of GUI component properties. This approach is limited, given that it can inspect only a very small set of software accessibility requirements.

3) There are validation tools that inspect source code to evaluate accessibility. This approach is not feasible in the software case, given the difficulties of correctly interpreting programming languages.

Checklists only state the requirements to be met, but do not explain in detail how to evaluate these requirements. When human beings do the assessment, this lack of detailed procedures leads evaluators to put their own interpretation on things. This can end up in inconsistent results across a team of evaluators.

Finally, as the research on accessibility progresses, existing sets of guidelines are updated and new sets are proposed by diverse institutions. These guidelines may be inconsistent with other guidelines. For this reason, tools should be provided to manage guidelines, as proposed for the web domain.

After surveying the state of the art in software accessibility assessment, we find that there is no broadly accepted method ।.

\section{Requirements for a Software Accessibility Conformity Assessment Method}

The paper will provide a set of requirements that should be taken into account when defining software accessibility conformity assessment methods. The discussion will be based on our experience in evaluating software accessibility and on the work that is being carried out in response to the European Commission's Mandate M376.

The ISO standards define a functional model for conformity assessments, based on the following four functions

1. Selection of the object of assessment (software in our case), the requirements and the methods for performing the other functions.

2. Determination to gather full information regarding fulfilment of the specified requirements by the object of the conformity assessment or a sample thereof.

3. Review and Attestation, consisting of final checking of the decision and the production of the statement of conformity. 
4. Surveillance, that is, systematic iteration of the assessment activities to assure the validity of the statement. Surveillance is optional and may be needed, for instance, when the object of conformity changes over time.

\subsection{Selection}

Selection involves planning and preparation activities to gather or produce all the information and input needed for the subsequent determination function This includes selection of the object of conformity assessment (sampling may be necessary to select a part of the entire object that is representative of the whole), consideration of the specified requirements and choice of the most appropriate procedures to be used for determination activities. The requirements we suggest for selection are:

- Selection of the object of conformity assessment

- The method should provide guidance about how to divide a software product into separate and simpler elements (user tasks, functions, steps, etc.) for evaluation. Typically software products are complex and, given that each requirement is typically determined for each individual element, need to be divided to facilitate assessment process planning. In previous work we divided desktop operating systems (OS) into two main groups of functionality (task and file management), each with several user tasks (like get a list of running tasks, task switching, or elemental file operations). We then evaluated each requirement for each individual user task.

- The method should also define sampling techniques to be used when the software product is too complex to be evaluated exhaustively. In complex software products (such as the OS evaluated in ) it is unfeasible, due to time and budget constraints, to fully evaluate the accessibility of each individual element. In these cases the method should drive the sampling process to guarantee that the final result of the assessment is representative enough.

- The method should include tool support for decomposing the software product into individual elements and applying sampling techniques (if required) in order to ease and systematize the creation of a list of features to be evaluated during the conformity assessment.

- Selection of requirements

- The list of requirements should be based on international software accessibility standards to improve the applicability of the results in different contexts, to foster international harmonization and to minimize trade barriers The recommended standard for software accessibility is the upcoming ISO 9241-171 described above. In our previous work we used a preliminary version of this document, ISO TS 16071 , plus some requirements from Spanish standard UNE 139802 , because ISO 9241-171 was unavailable at the time. The combined content of the other two standards covers most of the future standard's recommendations.

- The method should allow for the resulting checklist to be tailored to different stringency levels. ISO 9241-171, for instance, has two levels: requirements and recommendations A method for software accessibility conformity assessment should 
provide guidance for determining the applicability of requirements and recommendations to a given situation and for choosing the set of checkpoints that best suits the objectives of the conformity assessment. In our previous work, we chose to evaluate only the top-level requirements from the combination of ISO TS 16071 and UNE 139802. This generated a checklist of 39 items

- The method should include tool support for the management of the checklist, including the addition or removal of selected checkpoints based on ISO 9241-171 requirements and recommendations.

The selection of procedures to be used for determination activities will be discussed in the next section, focusing on the determination function.

\subsection{Determination}

Determination activities are undertaken to generate comprehensive information regarding the fulfilment of the specified requirements by the object of conformity assessment or a sample thereof. Some examples of determination activities are testing, inspection, auditing and peer assessment [4]. In the case of software accessibility assessment the most used types of determination activities are testing (by expert users) and inspection. The requirements that we suggest for the determination function are:

- The method should precisely define how to evaluate each of the checkpoints to assure maximum assessment objectivity and repeatability. In our previous work we observed a high degree of variability in results across different evaluators when directly evaluating the checkpoints without a detailed testing procedure This variability could not be explained either by their previous knowledge of the two desktop OS or by the order in which they did the assessment.

- The method should also clearly state the possible values of the evaluation of each of the checkpoints. In our previous work we used a set of five possible values: pass, fail, partial, unknown and not applicable which are considered to be sufficient for the task of accessibility assessment.

- The method should include tools to help evaluators to assess specific requirements. Some existing examples are for the evaluation of colour contrast and for the evaluation of screen flickering.

- The method should include tool support for recording ratings for each of the checkpoints applied to each of the individual software elements. This information will be used in the next stage (review and attestation) to produce the final result of the conformity assessment. Hera is an example of such a tool for the web context.

- The method should provide support for workgroup-based assessment. Given the amount of time it takes to assess software accessibility, the determination will in many cases be performed by teams of expert evaluators. Accordingly, the method should provide support for dividing the workload between team members and for managing the progress made by the different evaluators, and the method should include tool support for managing these workgroups. 


\subsection{Review and Attestation}

Review constitutes the final stage of checking before taking the important decision as to whether or not the object of conformity assessment has been reliably demonstrated to fulfil the specified requirements. Attestation results in a statement in a form that most readily reaches all of the potential users. The requirements we suggest for this function are:

- Review

- The method should detail how to aggregate the results of evaluating separate requirements and overall assessments in order to provide support for the final review process and the decision about the accessibility conformity of the software. In our work we presented a simple mechanism, based on the idea of prioritizing the worst results: if any individual element of the software is not accessible, then the software is considered not to be accessible. Another more sophisticated example of results aggregation has been presented in UWEM . It takes into account the selection phase sampling and can produce an aggregated numerical result. This aggregation of results should be defined for individual and for workgroup-based assessments.

- The method should incorporate tool support for aggregating the results. This tool could also detect inconsistencies found in the results that could be used to analyse the performance and reliability of the members of an assessment workgroup. This is one of the lines of future work that we identified in the case of web accessibility evaluation

- Attestation

- The method should define how to provide a detailed attestation, containing both the aggregated result and the particulars of the evaluation of each checkpoint for each of the individual elements of the software product. The goal of such a report is not only to provide the final result of the assessment, but also to supply detailed information that developers can use to improve the product, or public procurers can apply to choose the most accessible product from a set of tenders. Examples of these reports are defined in the web context for UWEM and Hera Note also that the method should define templates for more or less detailed human-readable reports, from full reports to summaries.

- The method should also enable the use of a machine-readable semantic language to represent the detailed results of the assessment. This type of representation could be used by software to make automatic comparisons of the accessibility of several software products or to compare the results generated by two different evaluators. An example of such a semantic language for the web context is EARL (Evaluation and Report Language) . This language could be adapted and extended to represent software accessibility reports.

- Finally, the method should include tool support for the automatic generation of both human-readable and machine-readable reports, using the information gathered throughout the whole assessment process. This would ease the task of reliably communicating the results. 


\subsection{Surveillance}

Conformity assessment can end after attestation. However, in some cases, systematic iteration of the assessment functions may be needed to assure the validity of the statement resulting from attestation. The user needs drive such activities. For example, an object of conformity assessment may change over time. This could affect its continuing fulfilment of specified requirements. The requirements that we suggest for the surveillance function are:

- The method should provide support for decision making on whether a software product requires surveillance or not This decision is typically based on the rate of change. One good example is current operating systems with auto-update features, making it necessary to reiterate the assessment to assure the validity of the attestation.

- The method should also provide support for determining the rate of surveillance activities and for dealing with changes in the assessment result, especially in the cases when the statement has to be suspended or withdrawn

\section{Conclusions and Future Work}

Currently there is a need for rigorous methods for assessing software accessibility conformity against formal standards. As our experience has shown evaluators directly applying the guidance in these standards do not always get the same results, and there is likely to be inconsistency or uncertainty.

In this paper we have presented a set of requirements for a software accessibility conformity assessment method that minimizes this problem. The requirements apply across the four conformity assessment functions defined by ISO/IEC 17000: selection, determination, review and attestation, and surveillance. Most of the requirements we presented are the result of our previous experience in the field of accessibility assessment. Our future work is to develop and test a method and its accompanying tools based on these requirements to support the future demands of accessibility conformity assessment in the field of public procurement.

\section{References}

United States Department of Justice: 1998 Amendment to Section 508 of the Rehabilitation Act. Section 508 of the Rehabilitation Act (29 U.S.C. 794d), as amended by the Workforce Investment Act of 1998 (P.L. 105-220) (1998)

European Commission: Enterprise and Industry Directorate-General. Standardization mandate to CEN, CENELEC and ETSI in support of European accessibility requirements for public procurement of products and services in the ICT domain, M 376 - EN 2005-12-07 (2005)

ISO FDIS 9241-171. Ergonomics of human-system interaction - Part 171: Guidance on software accessibility. Final Draft International Standard. ISO (2008)

ISO/IEC 17000:2004, Conformity assessment - Vocabulary and general principles. International Standard. ISO/IEC (2004) 
World Wide Web Consortium: Evaluating Web Sites for Accessibility: Overview, http://www.w3 . org/WAI/ eval / Overview. html

Velleman, E., Strobbe, C., Koch, J., Velasco, C.A., Snaprud, M.A.: Unified Web Evaluation Methodology Using WCAG. In: Stephanidis, C. (ed.) HCI 2007. LNCS, vol. 4556, pp. 177-184. Springer, Heidelberg (2007)

Spanish Association of Standardization and Certification (AENOR): UNE 139802:2003. Computer applications for people with disabilities. Computer accessibility requirements. Software (in Spanish) (2003)

World Wide Web Consortium: Web Accessibility Initiative (WAI) - homepage, http: //Www.w3 . org/WAI/

Nielsen, J.: Usability inspection methods. In: Conference companion on Human factors in computing systems, Boston, Massachusetts, United States, April 24-28, pp. 413-414 (1994)

Feigenbaum, B., Squillace, M.: Accessibility Validation with RAVEN. In: Proceedings of the 2006 International Workshop on Software Quality, pp. 27-32. ACM, New York (2006) Abascal, J., Arrue, M., Fajardo, I., Garay, N., Tomás, J.: The use of guidelines to automatically verify Web accessibility. Universal Access in the Information Society 3(1), 7179 (2004)

Hideki Tanaka, E., Bim, S.A., Vieira da Rocha, H.: Comparing accessibility evaluation and usability evaluation in HagáQuê. In: Proceedings of the 2005 Latin American conference on Human-computer interaction. ACM International Conference Proceeding Series, Mexico, vol. 124, pp. 139-147 (2005)

González, A.L., Mariscal, G., Martínez, L., Ruiz, C.: Comparative Analysis of the Accessibility of Desktop Operating Systems. In: Stephanidis, C. (ed.) HCI 2007. LNCS, vol. 4554, pp. 676-685. Springer, Heidelberg (2007)

European Information \& Communications Technology Industry Association: EICTA White paper on eAccessibility (October 19, 2005),

http: / /www.eicta.org/web/news/telecharger.php?iddoc=374

International Standards Organization: ISO/TS 16071:2003. Ergonomics of human-system interaction - Guidance on accessibility for human-computer interfaces. Technical Specification (2003)

The Paciello Group: Contrast Analyzer, Version 2.0,

http://www.paciellogroup.com/resources/

contrast-analyser.html

Trace Center: Photosensitive Epilepsy Analysis Tool, http: //trace.wisc.edu/peat/

Benavidez, C., Fuertes, J.L., Gutiérrez, E., Martínez, L.: Semi-automatic Evaluation of Web Accessibility with HERA 2.0. In: Miesenberger, K., Klaus, J., Zagler, W., Karshmer, A.I. (eds.) ICCHP 2006. LNCS, vol. 4061, pp. 199-206. Springer, Heidelberg (2006)

World Wide Web Consortium: Evaluation and Report Language (EARL) 1.0 Schema (Working draft), http://WWW.W3 . org/TR/EARL10/ 